\title{
Enabling proximity mask-aligner lithography with a 193nm CW light source
}

Raoul Kirner, Andreas Vetter, Dmitrijs Opalevs, Matthias Scholz, Patrick Leisching, et al.

Raoul Kirner, Andreas Vetter, Dmitrijs Opalevs, Matthias Scholz, Patrick Leisching, Toralf Scharf, Wilfried Noell, Carsten Rockstuhl, Reinhard Voelkel, "Enabling proximity mask-aligner lithography with a 193nm CW light source," Proc. SPIE 10587, Optical Microlithography XXXI, 105871F (20 March 2018); doi: 10.1117/12.2297171

SPIE. Event: SPIE Advanced Lithography, 2018, San Jose, California, United States 


\title{
Enabling proximity mask-aligner lithography with a $193 \mathrm{~nm} \mathrm{CW}$ light source
}

\author{
Raoul Kirner ${ }^{*}, \mathrm{a}$, Andreas Vetter ${ }^{\mathrm{a}, \mathrm{b}}$, Dmitrijs Opalevs ${ }^{\mathrm{c}}$, Matthias Scholz ${ }^{\mathrm{c}}$, Patrick Leisching ${ }^{\mathrm{c}}$, \\ Toralf Scharf ${ }^{\mathrm{d}}$, Wilfried Noella ${ }^{\mathrm{a}}$, Carsten Rockstuhl ${ }^{\mathrm{b}, \mathrm{e}}$, and Reinhard Voelkel ${ }^{\mathrm{a}}$ \\ ${ }^{a}$ SUSS MicroOptics SA, Rouges-Terres 61, CH-2068 Hauterive, Switzerland \\ binstitute of Nanotechnology (INT), Karlsruhe Institute of Technology, \\ Hermann-von-Helmholtz-Platz 1, 76344 Eggenstein-Leopoldshafen, Germany \\ ${ }^{c}$ TOPTICA Photonics AG, Lochhamer Schlag 19, 82166 Gräfelfing, Germany \\ ${ }^{d}$ Nanophotonics and Metrology Laboratory, École Polytechnique Fédérale de Lausanne \\ (EPFL), CH-1015 Lausanne, Switzerland \\ eInstitute of Theoretical Solid State Physics (TFP), Karlsruhe Institute of Technology, \\ Wolfgang-Gaede-Strasse 1, 76131 Karlsruhe, Germany
}

\begin{abstract}
We introduce a novel industrial grade $193 \mathrm{~nm}$ continuous-wave laser light source for proximity mask-aligner lithography. A diode seed laser in master-oscillator power-amplification configuraton is frequency-quadrupled using lithiumtriborate and potassium-fluoro-beryllo-borate non-linear crystals. The large coherence-length of this monomodal laser is controlled by static and rotating shaped random diffusers. Beam shaping with imaging and non-imaging homogenizers realized with diffractive and refractive micro-optical elements is compared in simulation and measurement. We demonstrate resolution patterns offering resolutions $<2 \mu \mathrm{m}$ printed with proximity gaps of $20 \mu \mathrm{m}$.
\end{abstract}

Keywords: Diode lasers, laser beam shaping, frequency doubled lasers, UV lasers, lithography, microstructure fabrication

\section{INTRODUCTION}

Since the 1960's, mask-aligners (MAs) rely on high-pressure mercury arc lamp light sources. To overcome the limit imposed by their wavelength range (down to i-line, $365 \mathrm{~nm}$ ), the introduction of excimer laser sources (KrF@ @ $248 \mathrm{~nm}, \mathrm{ArF} @ 193 \mathrm{~nm}$ ) has been studied, but deemed problematic due to their properties of being bulky, complex, expensive, and requiring regular maintenance. ${ }^{1}$ We present a novel approach by introducing an industrial grade continuous-wave (CW) $193 \mathrm{~nm}$ laser light source for proximity MA lithography.

The resolution in proximity mode, the preferred mode of MA lithography in production environments, ${ }^{2}$ depends on the exposure wavelength $\lambda$ and the distance between photomask and substrate, the proximity gap $g$, following the relation Resolution $\propto \sqrt{\lambda g} .{ }^{3}$ As proximity gaps are generally limited to some $20 \mu \mathrm{m}$ to $50 \mu \mathrm{m}$, mainly due to substrate flatness and possible structures on the substrates, a reduction in exposure wavelength offers a possibility to decrease printable feature size.

In our light source, a laser diode emitting at $772 \mathrm{~nm}$ is amplified by a tapered amplifier in master-oscillator power-amplification (MOPA) configuration ${ }^{4,5}$ (TOPTICA PHOTONICS, DL pro). Frequency quadrupling in subsequent enhancement cavities using lithiumtriborate (LBO) and potassium-fluoro-beryllo-borate (KBBF) non-linear crystals generates monomodal continuous emission at $193 \mathrm{~nm}$ with up to $20 \mathrm{~mW}$ optical output power. The laser light source features motorized mirror mounts enabling drop shipment and easy installation, with automatic mirror alignment. A motorized crystal shifter for the KBBF crystal enables an overall lifetime of the laser light source of up to $10000 \mathrm{~h}$.

\footnotetext{
*Further author information:

E-mail: raoul.kirner@suss.com, Telephone: +41 325664474 
MA lithography relies on uniform illumination with a uniform angular spectrum of the whole exposure field during exposure (usually equal to the size of the substrate). Periodic microoptical elements are well-suited for illumination homogenization. Such elements, realized as diffractive optical elements (DOEs) or microlens arrays (MLAs), subdivide an incident wavefront: each individual aperture transmits the part of the wavefront it sees, locally directed along its individual optical axis. A subsequent macroscopic lens combines the contributions of all individual channels in its focal plane, hence called Fourier lens. A second lens with identical focal length and sufficient clear aperture placed in the back-focal plane of the Fourier lens renders the achieved flat-top illumination telecentric, thus its denomination as field lens. This principle has been described in literature already, e.g. by Voelkel et al. ${ }^{6}$ or by Dickey. ${ }^{7}$

To enable MA lithography with our laser source, we investigate beam-shaping with non-imaging and imaging homogenizers. The non-imaging homogenizer is realized with an eight-level DOE in Fresnel configuration, while the imaging homogenizer features double-sided refractive MLAs. Advantages and disadvantages of both possibilities are discussed. To achieve telecentric illumination with a uniform intensity distribution and a uniform angular spectrum in the photomask plane, two homogenizers can be staged, one illuminating the other. ${ }^{8}$ The angular spectrum can be set by simple means of inserting mechanical apertures that limit the illuminated area of the second integrator, so-called illumination filter plates (IFPs), enabling shaped illumination. In this contribution the illumination setups are discussed with respect to achieved uniformity and efficiency, both in simulation and measurement. Wavefront-mixing of the microoptical elements in combination with the introduction of a combination of static and rotating shaped random diffusers (SRDs) addresses the problem of interference effects arising from the highly coherent laser light with a coherence length of $>300 \mathrm{~m} .{ }^{5}$ Together with the integration over time in the deep ultraviolet (DUV) photoresist, the arising speckles are effectively mitigated during exposure. ${ }^{6}$

Using the $193 \mathrm{~nm}$ diode laser source with these beam shaping variants, we report on the resolution and quality of lines and spaces (LS) patterns printed in proximity mode with a standard binary photomask. We demonstrate structures offering a resolution better than $2 \mu \mathrm{m}$ for proximity gaps of $20 \mu \mathrm{m}$.

\section{BEAM-SHAPING FOR MASK ALIGNER LITHOGRAPHY}

MA lithography relies on uniform illumination of the full exposure field. For the $193 \mathrm{~nm}$ CW laser as light source also the degree of coherence has to be decreased to avoid intensity variations caused by interference patterns in the exposed photoresist. The coherence length has to be made smaller than the smallest distance in the optical setup to suppress visible interference fringes. Here, two setups are compared: a non-imaging homogenizer setup featuring a DOE as the mixing element and an imaging homogenizer featuring double-sided MLAs. Figure 1a shows the overall beam path in our test setup as well as the two investigated beam-shaping optical setups.

Figure 1b shows a non-imaging homogenizer setup similar to the setup as realized in prior work. ${ }^{9}$ The laser beam, incident from the top, passes a combination of first a static and second a rotating SRD, called SD and RD in the figure. The expanding beam is then roughly collimated by lens $\mathrm{L}_{\mathrm{C}}$ to ensure fully developed speckle incident on the DOE. This collimation is possible due to the nature of the SRD. ${ }^{9}$ Size and shape of the illuminated area of the DOE is set by means of a mechanical aperture, the IFP, placed just before the element. The illuminated area defines the angular spectrum of the illumination in the mask plane, once all channels' contributions are superimposed by the Fourier lens $\mathrm{F}_{\mathrm{FT}}$. Telecentric illumination in the mask plane is ensured by the field lens $\mathrm{F}_{\mathrm{FL}}$. A particularity of this setup is the zero order blocking filter (ZOBF): an opaque aperture placed on the optical axis close to the superposition plane to suppress the strong zero order, caused by imperfections of the DOE. Placing the ZOBF slightly out of focus of the flat-top illumination plane attenuates the zero order, thus enabling uniform exposure of the whole field. To illuminate an exposure field slightly bigger than $1 \mathrm{~cm}^{2}$, Fourier and field lens with a focal length of $200 \mathrm{~mm}$ were chosen. A relay lens system can be used to increase the distance between the homogenizer setup and the mask plane, if necessary. ${ }^{9}$

The setup shown in Fig. 1c depicts an imaging homogenizer setup, realized with a double-sided MLA (For 1D homogenization, a cylinder lens array as shown is sufficient. For 2D homogenization, two crossed cylinder lens arrays, both placed in close mutual proximity, are required). The cylinder lens array is manufactured in a way that front and back side lens possess the same focal length, which furthermore is equal to the element thickness. This means that the back-side array images the entrance pupil of the first array to infinity, hence its description 


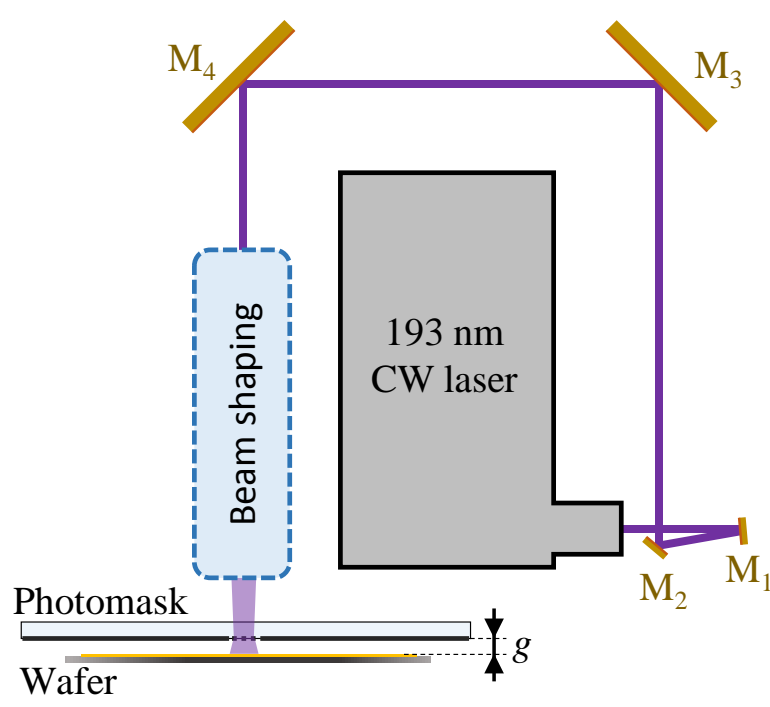

(a)

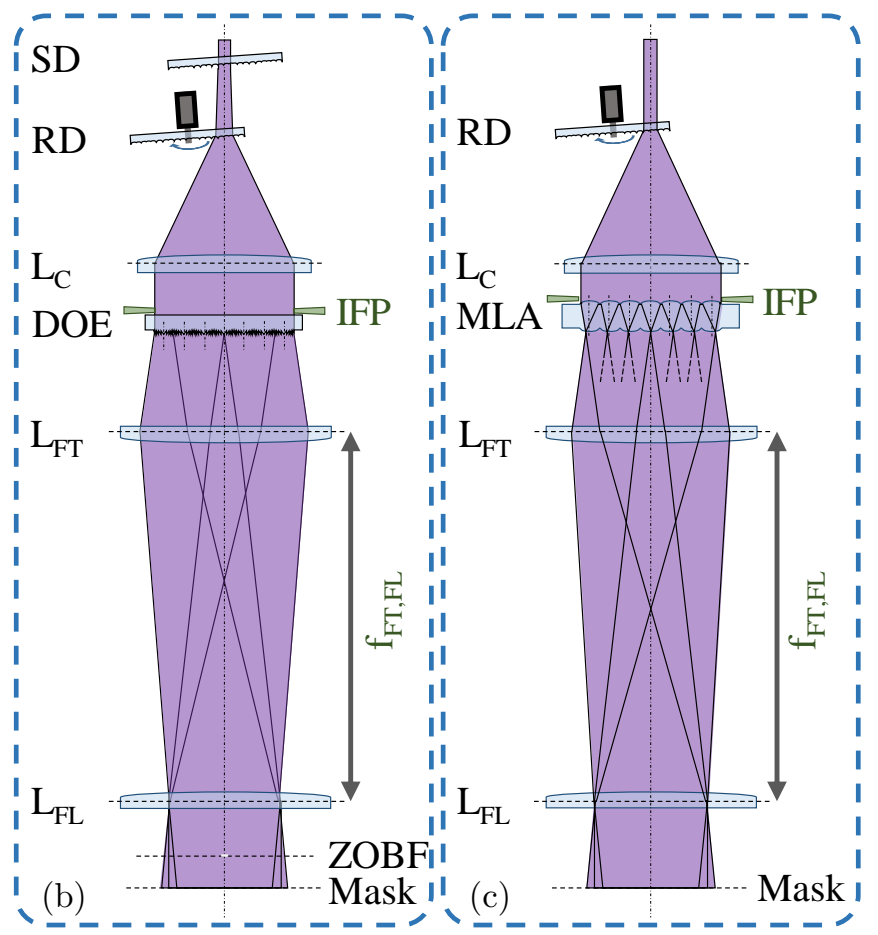

Figure 1: Exposure setup as realized for mask aligner lithography testing with the $193 \mathrm{~nm}$ laser(a). The laser beam is redirected with mirrors $\mathrm{M}_{1-4}$ to the photomask, passing optical elements for beam shaping. (b) Non-imaging homogenizer beam-shaping setup with DOE as described in 9. (c) Imaging homogenizer beam-shaping setup with MLA. Both setups feature beam homogenizing with microoptical multi-aperture elements for subdivision of an incident wavefront with subsequent superposition. Mechanical apertures, named illumination filter plates (IFP), allow to control the angular spectrum in the mask plane. All other abbreviations are given in the text. Schemata are not to scale.

as imaging homogenizer. This geometrical layout renders the element tolerant against angled illumination (in limits given by the lens parameters). Subaperture superposition is realized with a Fourier lens and telecentricity is ensured by a field lens, as described for the non-imaging setup. Due to the smaller pitch of subapertures in case of the MLA compared to the DOE, illumination mixing is more effective by a factor of approximately 25 in the case shown here. Thus only one diffuser is needed for speckle mitigation. Nevertheless, an element variant in time, here the rotation of the diffuser, is needed, to suppress the visibility of speckle in the photoresist.

\subsection{Optical simulation of an imaging homogenizer setup}

Similar to the simulation of a non-imaging beam-shaping setup with the $193 \mathrm{~nm}$ CW laser ${ }^{9}$ imaging homogenizer setups can be modeled and simulated. All simulations have been made with the commercial ray-tracing software ZEMAx OpticsStudio. Rotating diffusers and time-averaging in the photoresist allow for incoherent simulation. Figure 2 shows the results of a simulation of an imaging homogenizer setup as depicted in Fig. 1c. Two crossed double-sided cylinder lens arrays (Fused Silica, uncoated) with lens pitch of $300 \mu \mathrm{m}$ and a maximum acceptance angle of $6^{\circ}$ were used to realize the micooptical mixing element. Fused Silica singlet lenses of $72.5 \mathrm{~mm}$ focal length at $193 \mathrm{~nm}$ were used as Fourier and field lens. With this combination an exposure field of $15 \mathrm{~mm}$ by $15 \mathrm{~mm}$ is realized, compare Fig. 2a. The cross-shaped artifact visible in this figure stems from the flat surface area in between the lenslets of the MLAs. IFPs, simulated as apertures limiting the irradiated area of the integrating element, control the angular spectrum in the mask plane. As the illumination of the integrator is not uniform in our case, but rather given by the central part of the beam profile after the diffuser, the angular spectrum shows the same (Gaussian) characteristics. This can be seen in Figs. 2b and 2c for two different IFPs. 

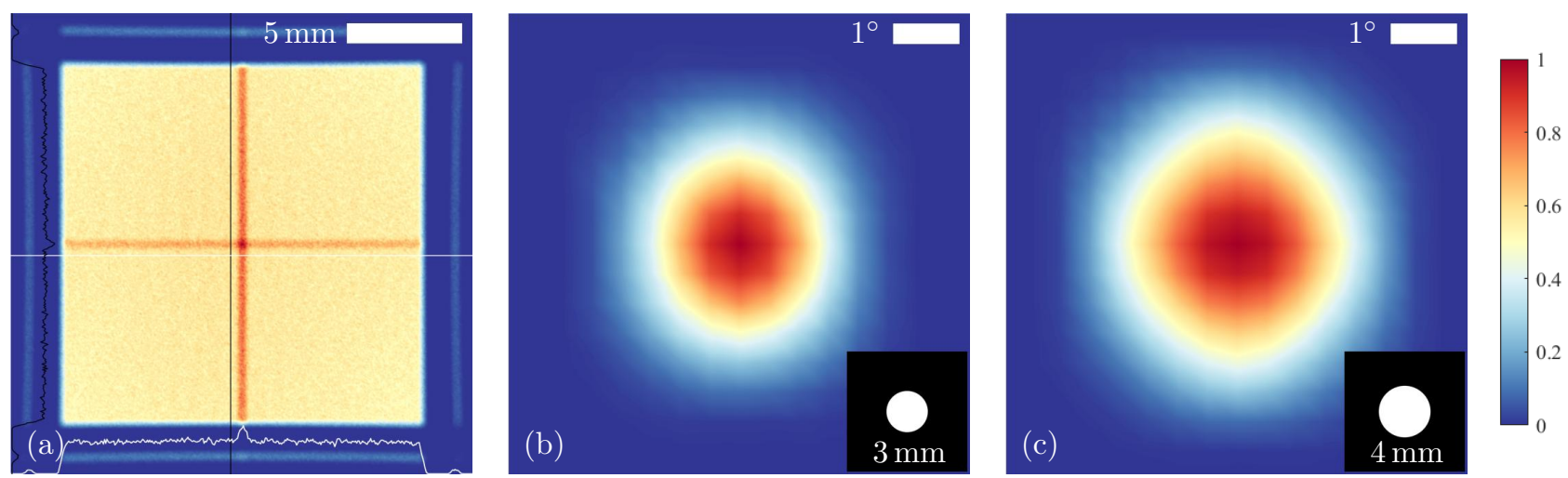

Figure 2: Simulation of the illumination in the mask plane with an imaging homogenizer beam-shaping setup, as described in Fig. 1c. (a) shows the intensity distribution, while (b) and (c) show simulations of the angular spectrum in the mask plane (after one Koehler integrator stage) for IFPs with free diameters of $3 \mathrm{~mm}$ and $4 \mathrm{~mm}$, respectively. The insets schematically depict the IFP sizes. The cross-shape visible over the whole illuminated area in (a) stems from artifacts in the modeling of the MLA.

In general it is preferred to have a uniform angular spectrum for MA lithography. To improve the uniformity of the angular spectrum, a second homogenizer stage could be placed in between diffuser and the integrator, illuminating the IFP, thus realizing a MO Exposure Optics ${ }^{\circledR}$ setup. ${ }^{8,10}$ For this proof-of-principle work, with the limited optical power provided by the laser light source, we choose to increase optical throughput by omitting the first integrator stage. We trade thus optical power in the mask plane against uniform angular spectrum. This does not limit the validity of our findings, it only highlights lithography's general demand for light.

\section{EXPERIMENTAL RESULTS}

The described beam-shaping techniques have been applied to an experimental MA setup. Measurements of the achieved illumination are shown and compared to the simulations. Lithographic test prints made with imaginghomogenizer illumination are shown to supplement and complete the investigation of $193 \mathrm{~nm}$ MA lithography with non-imaging homogenizer illumination by Kirner et al. ${ }^{9}$

\subsection{Beam homogenization}

To measure intensity distribution and angular spectrum in the mask plane, an adapted beam-profiler was used. It features a UV-sensitive Si photodiode (Hamamatsu S1226-18BQ) mounted on an automated precision XY-stage. A laser-cut aperture of $300 \mu \mathrm{m}$ x $300 \mu \mathrm{m}$ was used to define the measurement area of the photodiode.

Figure 3 shows measurements of the illumination in the mask plane. The imaging homogenizer setup achieves intensity uniformity better than $3 \%$ with a rotating diffuser over the full required exposure field of $1 \mathrm{~cm}^{2}$, see subfigure a). Compared to the simulation (Fig. 2a) one finds both results in good agreement. The cross-shaped artifact visible in the simulation is not visible, though, in the measurement. Contrary to the simulation model of the MLA, the inter-lens surface of manufactured cylinder lens arrays is not flat. Hence, it diffuses incident light, rather than propagating unchanged to the mask plane. This improves the illumination uniformity in a real setup. When the diffuser is static, an arbitrary speckle field can be observed, see Fig. 3c. The temporal integration over many statistically uncorrelated speckle fields in the photosensitive element (i.e. beam-profiler or photoresist) reduces the visibility of speckles and hence their effect on an exposure.

The angular spectra for IFPs with free diameters of $3 \mathrm{~mm}$ and $4 \mathrm{~mm}$ are shown in Figs. 3b and 3e, respectively. As the diffuser does not widen the laser beam to the full acceptance angle of a single channel of the micro-optical integrator, the individual channels are visible as dots. The Gaussian beam profile of the laser light source can be observed superimposed onto the total angular spectrum, as it is expected from simulation.

Figures $3 \mathrm{c}$ and $3 \mathrm{f}$ show measurements of the mask plane illuminated with a non-imaging illumination setup as depicted in Fig. 1b and investigated in Ref. 9, without and with ZOBF, respectively. The improved uniformity 

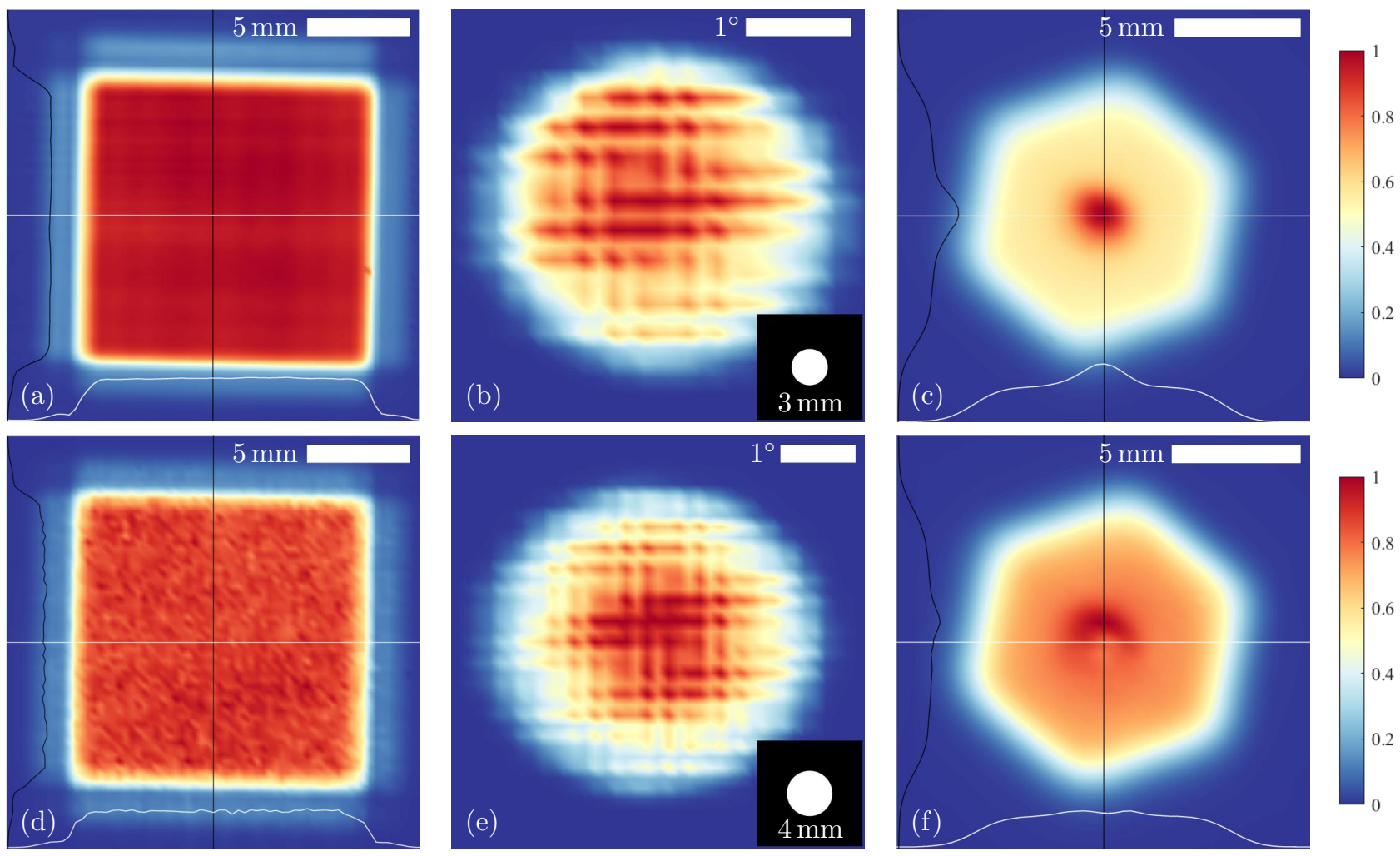

Figure 3: Measurement of the illumination profile in the mask plane. Irradiance distribution with the imaging homogenizer beam-shaping setup and rotating diffuser(a) and with static diffuser (d). (b) and (e) show measurements of the irradiance/angle for different IFPs with different free diameters, as stated in the inset pictures. (c) and (f) show the irradiance distribution in the mask plane as achieved with a non-imaging setup after 9, compare Fig. 1b, without and with ZOBF, respectively.

with ZOBF in Fig. $3 \mathrm{f}$ is clearly visible, while some remainders of the zero order are still visible. These are caused by non-ideal placement of the ZOBF. This directly illustrates one drawback of the DOE as microoptical mixing element, namely its limited efficiency.

In terms of illumination uniformity in the mask-plane, the imaging homogenizer as realized here clearly outperforms the non-imaging homogenizer setup, compare Figs. 3a and 3f. This conforms to our expectations, which can also be found in literature, ${ }^{6,7}$ as the imaging homogenizer superimposes the contributions of all subapertures onto the same field indifferently to the propagation direction of the incident wavefront. In comparison, the nonimaging homogenizer only perfectly superimposes all contributions in case of an overall plane wave illumination, which is not the case here after the diffuser.

Yet another thought on the shape of the exposure field: the crossed cylinder lens arrays used for the imaging homogenizer generate a square exposure field due to their square subapertures. The DOE featured in the nonimaging setup with its hexagonal lenslets shapes the exposure field similarly. Both shapes can be preferable, depending on the application.

\subsection{Photolithographic prints}

To demonstrate the viability of the imaging homogenizer beam-shaping setup for mask-aligner lithography with the novel $193 \mathrm{~nm}$ CW laser light source, printing tests were conducted. The illumination setup was integrated into our versatile MA testbench featuring an ETEL SARIGAN* high-precision movement stage for wafer positioning.

*ETEL S.A., CH-2112 Môutier, Switzerland, etel.ch, etel@etel.ch 

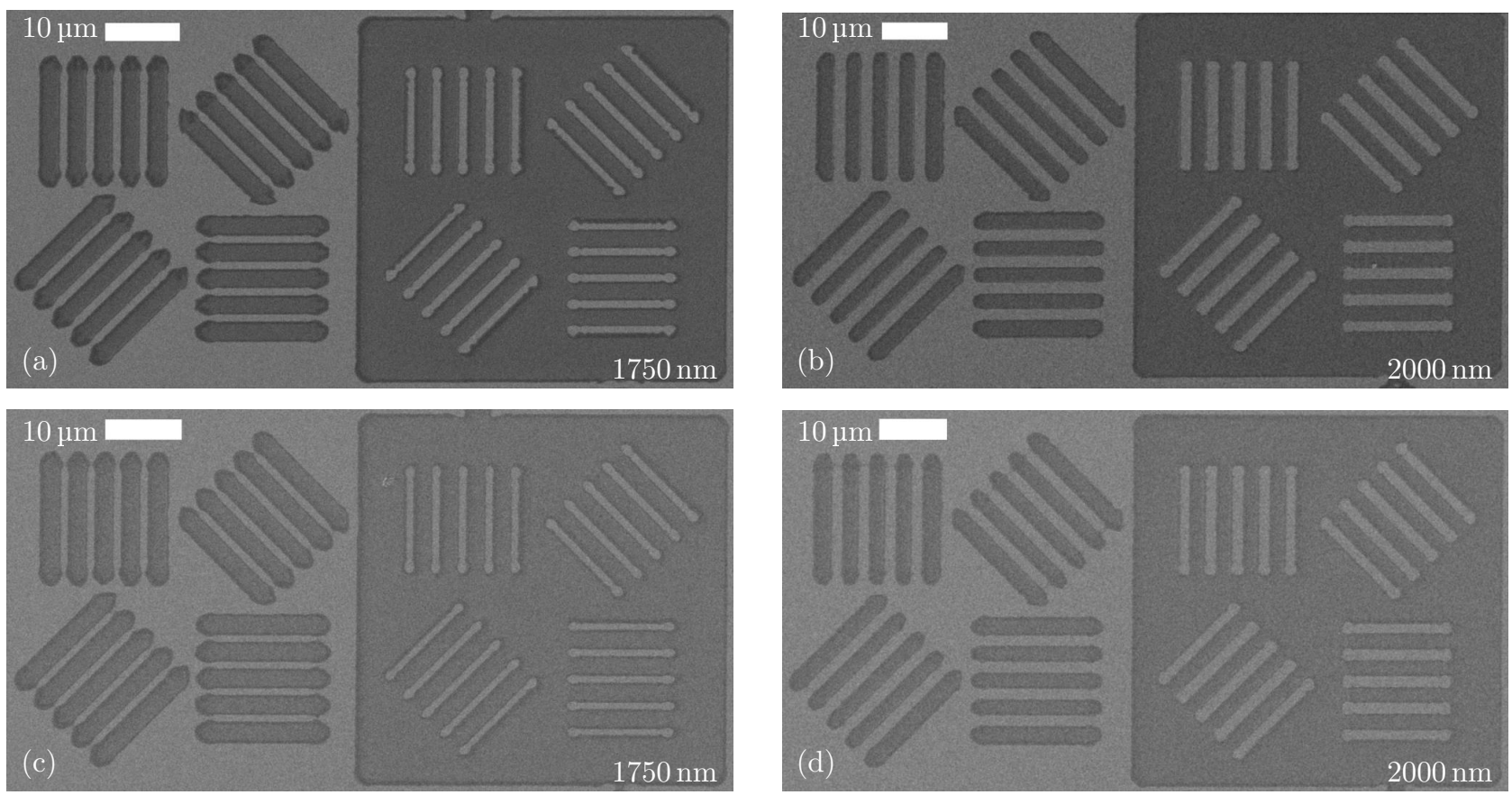

Figure 4: Micrographs of test prints realized with the imaging homogenizer illumination setup. All prints show LS resolution structures printed into $120 \mathrm{~nm}$ TOK TARF-P6239 photoresist with $20 \mu \mathrm{m}$ proximity gap. (a) and (b) were printed with an IFP of $3 \mathrm{~mm}$ diameter while (c) and (d) were printed with an IFP of $4 \mathrm{~mm}$ diamter, compare Fig. 3. The inset number in the lower right corner gives the half-pitch as minimum feature size of each group of LS. (a) and (b) as well as (c) and (d) show structures from the same exposure on the same wafer, respectively.

An Avantes ${ }^{\dagger}$ spectroscopic reflectometry setup was used to precisely set and control proximity gaps of $20 \mu \mathrm{m}$. All prints were made on Silicon wafers spincoated with a $120 \mathrm{~nm}$ layer of the DUV photoresist TOK TARF-P6239 ME. This photoresist is optimized for lines and spaces (LS) patterns. Wafer processing included prebake and post-exposure bake on a hotplate each of $60 \mathrm{sec}$ at $110^{\circ} \mathrm{C}$. The wafers were developed for one minute in $\mathrm{AZ}^{\circledR}$ MIF 327 in a puddle process. The exposure dose was set to $45 \mathrm{~mJ} \mathrm{~cm}^{-2}$.

Figure 4 shows Scanning Electron Microscope (SEM) micrographs of lines and spaces(LS) structures printed with IFPs of $3 \mathrm{~mm}$, a) and b), and $4 \mathrm{~mm}$, c) and d). We repeatedly achieved sub-2 $4 \mathrm{~m}$ resolution, for different IFP configurations, as shown in a) and c), with linewidths shown of $1.75 \mu \mathrm{m}$. Visible in all prints are line-end problems, which are expected for proximity gaps of $20 \mu \mathrm{m}$. One can also observe the dependency of the exposure dose on the feature size of interest. The presented prints were exposed with doses set for $2 \mu \mathrm{m}$ structures, whereas the $1.75 \mu \mathrm{m}$ structures are still underexposed. To omit the line-end problems, optical proximity correction (OPC) structures could be implemented in the photomask, which was not within the scope of this work.

\section{CONCLUSION \& OUTLOOK}

In this contribution we investigated the viability of and the necessary beam-shaping for MA lithography in proximity mode with a novel industrial grade $193 \mathrm{~nm} \mathrm{CW}$ laser light source. This work constitutes an addition and extension to the work presented in the Optics ExpREss Journal by the authors. ${ }^{9}$

An imaging homogenizer beam-shaping setup, realized with crossed cylinder-lens MLAs as micro-optical mixing elements, was investigated. The setup was simulated with commercial optics simulation software and compared to measurements, both were found to be in good agreement. Furthermore, the production process of

\footnotetext{
†Avantes BV, NL-7333 NS Apeldoorn, The Netherlands, avantes.com, info@avantes.com
} 
the MLA enhances the actual performance of the homogenizer by suppressing artifacts caused by inter-lens areas. Imaging and non-imaging homogenizer setups, the latter realized with a DOE as micro-optical mixing element, were discussed and compared. Imaging homogenizer setups are inherently insensitive to incoherent illumination in comparison to non-imaging homogenizers, rendering them more suited for beam mixing of speckle fields after e.g. a diffuser.

To show the viability of the presented illumination setup for MA lithography testprints were presented. Lines and spaces resolution structures printed into $120 \mathrm{~nm}$ DUV photoresist on silicon substrates with proximity gaps of $20 \mu \mathrm{m}$ exhibit sub-2 $\mu \mathrm{m}$ resolution.

We would like to emphasize once more the proof-of-principle character of the presented work. The shown results were achieved on small exposure fields, viable without restriction of generality, while the realization of larger exposure fields would require improved optical output power of the laser light source for exposure times on a realistic time scale.

One possible application for the presented MA setup with $193 \mathrm{~nm} \mathrm{CW}$ laser light source with its current optical output power is Talbot MA lithography, as presented by A. Vetter et al. ${ }^{11}$ Here the application of smaller wavelengths, compared to classical $\mathrm{Hg}$ high-pressure arc lamp light sources, offers direct benefits for achievable resolution and process robustness.

\section{ACKNOWLEDGMENTS}

The authors want to thank S. Tournois and J. Béguelin for useful discussions and technical support. Furthermore, the authors acknowledge the preparation of KBBF crystals by R. Li, X. Wang, and L. Liu. A.V. acknowledges support by the Karlsruhe School of Optics and Photonics (KSOP).

This project has received funding from the European Union's Horizon 2020 research and innovation programme under the Marie Skłodowska-Curie grant agreement No 675745.

\section{REFERENCES}

[1] Partel, S., Zoppel, S., Hudek, P., Bich, A., Vogler, U., Hornung, M., and Voelkel, R., "Contact and proximity lithography using 193nm Excimer laser in Mask Aligner," Microelectronic Engineering 87(5-8), 936 (2010).

[2] Mack, C., [Fundamental Principles of Optical Lithography], John Wiley \& Sons, Ltd, Chichester, UK (2007).

[3] Rai-Choudhury, P., ed., [Handbook of microlithography, micromachining, and microfabrication. Volume 1: Microlithography], SPIE Optical Engineering Pr., Bellingham, Wash. (1997).

[4] Scholz, M., Opalevs, D., Leisching, P., Kaenders, W., Wang, G., Wang, X., Li, R., and Chen, C., "A bright continuous-wave laser source at 193 nm," Applied Physics Letters 103(5), 051114 (2013).

[5] Opalevs, D., Scholz, M., Gilfert, C., Li, R., Wang, X., Liu, L., Vetter, A., Kirner, R., Scharf, T., Noell, W., Rockstuhl, C., Voelkel, R., and Leisching, P., "Semiconductor-based narrow-line and high-brilliance 193nm laser system for industrial applications," Presented at SPIE Photonics West 2018, Paper 10511-83 (2018).

[6] Voelkel, R. and Weible, K. J., "Laser beam homogenizing: limitations and constraints," Proc. SPIE $\mathbf{7 1 0 2}$ (2008).

[7] Dickey, F. M., ed., [Laser Beam Shaping: Theory and Techniques], CRC Press (2017).

[8] Voelkel, R., Vogler, U., Bich, A., Pernet, P., Weible, K. J., Hornung, M., Zoberbier, R., Cullmann, E., Stürzebecher, L., Harzendorf, T., and Zeitner, U. D., "Advanced mask aligner lithography: new illumination system," Optics Express 18(20), 20968 (2010).

[9] Kirner, R., Vetter, A., Opalevs, D., Gilfert, C., Scholz, M., Leisching, P., Scharf, T., Noell, W., Rockstuhl, C., and Voelkel, R., "Mask-aligner lithography using a continuous-wave diode laser frequency-quadrupled to 193 nm," Optics Express 26(2), 730 (2018).

[10] Voelkel, R., Vogler, U., Bich, A., Weible, K. J., Eisner, M., Hornung, M., Kaiser, P., Zoberbier, R., and Cullmann, E., "Illumination system for a microlithographic contact and proximity exposure apparatus," European Patent 2253997 A2 (2009).

[11] Vetter, A., Kirner, R., Opalevs, D., Scholz, M., Leisching, P., Scharf, T., Noell, W., Rockstuhl, C., and Voelkel, R., "Mask-aligner Talbot lithography using a 193nm CW light source," Presented at SPIE Advanced Lithography 2018, Paper 10587-33 (2018). 